\title{
Accounting for variability in student responses to motion questions
}

\author{
Brian W. Frank \\ Department of Physics, University of Maryland, College Park, Maryland 20742, USA \\ Stephen E. Kanim \\ Department of Physics, New Mexico State University, Las Cruces, New Mexico 88003, USA
}

Luanna S. Gomez

Department of Physics, University at Buffalo (SUNY), Buffalo, New York 14222, USA

(Received 29 May 2007; revised manuscript received 27 May 2008; published 23 September 2008)

\begin{abstract}
We describe the results of an experiment conducted to test predictions about student responses to questions about motion based on an explicit model of student thinking in terms of the cuing of a variety of different physical intuitions or conceptual resources. This particular model allows us to account for observed variations in patterns of student responses in a way that positing the existence of fixed student conceptions about motion does not. As a result of the experiment, we suggest refinements of our model in order to better account for additional features of the student responses.
\end{abstract}

DOI: 10.1103/PhysRevSTPER.4.020102

PACS number(s): 01.40.Fk

\section{INTRODUCTION}

Physics education research is often conducted without explicit consideration of models of student thinking. Researchers design questions that are intended to explore student ideas about a topic, and then student responses are analyzed. Some common student responses suggest thought processes that may need correction or at least further investigation. What is masked by this procedure is that the search for common responses is often guided by implicit assumptions about what these responses tell us. For example, a student might write that a tossed coin moving upward has an upward force from the hand acting on it and that it takes a while for gravity to overcome the upward force from the hand. ${ }^{1}$ Based on the ubiquity of similar responses, a researcher might characterize this response as reflecting a belief (more careful researchers may state that the response is "consistent with a belief") that motion in a given direction requires a force in that direction. Implicit in this kind of characterization is the assumption that a student giving this response would respond to a similar question in a manner that is also consistent with this belief. An example of this attribution of stable beliefs or conceptions to students' responses is this remark by Halloun and Hestenes $^{2}$ appearing in the American Journal of Physics:

During the interviews, the students repeated the answers they had given on the written test virtually without exception. Moreover, they were not easily swayed from their answers when individual questions were discussed, and they were usually able to give reasons for their choices. It seemed clear to the interviewer that the students' answers reflected stable beliefs rather than tentative, random, or flippant responses (p. 1044).

In this paper we provide examples of questions for which we believe that incorrect responses do not reflect stable beliefs that students have about the physical situation. Rather, we believe that these responses are assembled from a variety of different intuitions and that this assembly largely occurs at the time that the students answer the question. To support this claim, we will use a statistical analysis of observed variation in the pattern of responses from groups of students when details of the questions asked are changed. We draw inferences about the thinking processes of individual students based on the changes we observe in the behavior of groups of students when the conditions are changed. The relationship between our model for student thinking and the behavior of the groups of students in our experiment is analogous to the relationship between models for the microscopic behavior of molecules during a collision and measurements of gas properties when macroscopic parameters are varied. Just as a model for the physics of interactions between molecules allows predictions to be made about how a gas will behave when pressure or temperature is varied, a claim that students are basing their responses on intuitions that are assembled in response to question cues provides a model on which we can make predictions about how responses will change when question particular change. We believe that our results can better be accounted for by our particular knowledge-in-pieces model than by any assumption about student conceptions or misconceptions about the physical situation.

\section{MOTIVATION FOR RESEARCH}

\section{A. Initial investigation}

Our investigation into student understanding of kinematics concepts originated as part of an NSF-funded project to develop research-based laboratory curriculum for algebrabased physics courses. ${ }^{3}$ As part of establishing a base line for this study, we administered a variety of conceptual surveys over the span of a few semesters at Arizona State University (ASU) aimed at measuring student understanding of concepts covered in a traditional laboratory on projectile motion. In this laboratory, students measured relevant distances, speeds, and times in order to verify that the equations of motion predicted by Newton's laws were satisfied for balls 
that were launched horizontally from a tabletop onto the floor.

Students were not explicitly asked to make qualitative comparisons between the outcomes for balls launched with different speeds. However, they were required to run the experiment for at least two different launch speeds. As part of performing the necessary calculations, it was assumed that students would notice that the time for the balls to reach the ground was independent of its initial velocity and that the horizontal distance traveled was proportional to it. In the postlaboratory surveys we administered, students were asked to compare the times taken to reach the ground. In some of the surveys it was given that the balls were launched with various speeds off the tabletop; other surveys indicated that the balls landed at various locations on the floor. In this paper, we will refer to these questions as speed-cuing tasks and distance-cuing tasks, respectively.

Previous research results have provided characterizations of students' thinking about trajectories that bear on how students might respond to these questions. For example, McCloskey, ${ }^{4}$ Clement, ${ }^{5}$ Halloun and Hestenes, ${ }^{6}$ and Champagne et al. ${ }^{7}$ all described students' thinking about this particular physical situation (a horizontally launched projectile) in terms of students having a naive theory or misconception about motion similar to various historical physical theories. In particular, McCloskey ${ }^{4}$ characterized students' answers to such questions as being based on a belief that a faster moving ball carries with it a greater impetus (internal force) to resist the pull of gravity. McCloskey ${ }^{4}$ used this impetustheory description in order to specifically account for students' incorrect answers concerning the shape of trajectories and not necessarily their answer concerning the time to fall. However, based on these characterizations, one might make some inferences about what incorrect answers to expect from students in response to time questions. In particular, we claim that if student responses are based on an impetus-like belief, then they should answer both versions of the question in the same way - that the ball traveling faster or farther takes more time to hit the ground (since carrying more impetus it allows the ball to resist gravity longer).

In the process of analyzing our students' responses, however, we found that the distributions of students' incorrect answers depended on which version of the question was asked. While a majority of the wrong answers on the distance-cuing version were that longer distances took more time, the incorrect answers on the speed-cuing task were evenly split between faster objects taking more time and faster objects taking less time. Even if one does not accept the particular account presented by McCloskey, ${ }^{4}$ it is difficult to understand how the nature of students' difficulties with this problem could stem from a single incorrect belief or misconception concerning the trajectory of falling objects. The variation in student responses suggests that the student thinking is not fixed upon a single incorrect idea and that the details of the questions influence how students were responding. It would seem, based on the data, that a different characterization of students' knowledge and thinking about motion is needed in order to account for the results.

\section{B. Alternative account}

An alternative account of students' thinking about the projectile motion question is one based on the idea that students have a variety of different physical intuitions to bring to bear upon the question. We propose a simple model of their knowledge in terms of three intuitions for thinking about motion which may be more or less productive depending on the specific context. For example, students are likely to have the intuition that going faster implies taking less time. While such an intuition can be applied appropriately to infer that the fastest runner in a hundred-meter dash will take less time to cross the finish line, it does not always follow that taking the highway (where you travel at a greater speed) takes less time than the back roads (which may be less distance). The intuition that going faster implies taking less time itself is neither right nor wrong but rather can be applied appropriately or inappropriately depending on the situation. This intuition as a piece of students' knowledge helps us to account for why a significant fraction of students answer that the fastest ball rolling off the table takes the least amount of time.

In addition to the intuition going faster implies taking less time, students are likely to have intuitions such as going farther takes more time and going faster implies getting farther. These three aspects of students' intuitions reflect an understanding of the interrelationships among distance, speed, and time. While any one of these intuitions may arise in students' thinking about motion to make an inference or draw a conclusion, they may also be combined together (consciously or unconsciously) to form a variety of different arguments. For example, in the projectile motion question, a student may think that going faster off the edge of table implies going farther. They may then additionally apply their intuition that going farther implies taking longer, leading to the ultimate conclusion that going faster off the edge of the table implies taking more time to hit the ground. While this answer is consistent with McCloskey's account ${ }^{4}$ of student thinking based on naive impetus theories, the basis for this answer in students' thinking is slightly different (although not necessarily incompatible).

It is always possible to characterize students' thinking in a variety of different ways. For the projectile motion question, we can interpret their responses as reflections of stable theories or beliefs that they have or as ad hoc assemblies of more simple intuitions. These two different characterizations provide different perspectives on students' thinking that may be more or less adequate for capturing particular features of students' responses. For the questions we describe in this paper, we find that characterizing students' knowledge as composed of a variety of different intuitions provides a means for understanding both the variability and the context sensitivity of students' responses.

In Sec. III we describe the theoretical framework that provides the basis for our model of student thinking. We then briefly present qualitative data illustrating how this model is used to interpret students' responses to a question about simple harmonic motion. Finally, we present the quantitative results of an experiment that was used to test model-based predictions concerning the variability of students' responses 
to questions when different cues were presented.

\section{THEORETICAL FRAMEWORK}

Accounts of students' thinking like the alternative one described above belong to a class of models that aim to characterize students' knowledge in terms of smaller pieces than are typically afforded by descriptions in terms of beliefs or conceptions. In the literature, these kinds of characterizations have been variously referred to as knowledge-in-pieces, resource-based, manifold, and fine-grain constructivist accounts. ${ }^{8}$ Some specific examples for describing students' intuitive knowledge in physics include diSessa's phenomenological primitives (p-prims), ${ }^{9}$ Minstrell's facets of knowledge, ${ }^{10}$ and Hammer's resources, ${ }^{11}$ each with their own set of theoretical properties and perspectives.

What is common to all of these frameworks is the manner in which they describe students' intuitive knowledge. Finegrained flexible pieces of knowledge are activated in networks and combined to form students' thinking about a specific question or situation. Responses to questions are constructed on the fly, assembled from pieces of knowledge that may consist of abstract minigeneralizations or contextspecific bits of knowledge for particular situations. A strength of this perspective is that it accounts for the possibility that students' ideas may not be self-consistent and that student responses may also be sensitive to minor changes in cues and contexts. Although they account for inconsistency, such frameworks do not rule out stability: common and stable patterns of thinking may emerge out of particular combinations of knowledge pieces that become reliably activated across many contexts.

diSessa's knowledge-in-pieces ${ }^{9}$ account of students' thinking about physical mechanisms in terms of p-prims is particularly relevant to the intuitions about motion we have described above. Examples of p-prims are not only notions such as dying away, balancing, and blocking but also intuitions such as going farther takes more time. This knowledge can be used appropriately or inappropriately in thinking about a variety of different physical situations. According to diSessa, ${ }^{9}$ the kinematical intuitions for thinking about speed, distance, and time relations are among the p-prims used by children. He stated that they are used by adults, as well, albeit with lower frequency and in more reliable ways. ${ }^{12}$ While a description of knowledge including these intuitions is quite consistent with literature describing children's developing conceptions of motion and speed, ${ }^{13}$ the extent to which they play a role in college students' thinking about physics questions involving motion is an empirical question.

While the three kinematical intuitions described above can be clearly understood and articulated in terms of p-prims, not all of the ideas students bring to bear to reason about physical situations are "p-prim-like." For example, later in this paper, we describe how the idea that gravity acts the same on all bodies affects student responses to another problem involving motion and gravity. This idea might be better regarded as a fact that students have taken away from instruction rather than as a part of their intuitive sense of mechanism. For this reason, we have chosen to simply use the term resource to describe an element of students' knowledge. We use the term resource broadly to include any knowledge that students use to help make an inference or draw a conclusion about the problems in our study. Since we will be referring to the kinematic intuitions that form our model quite often in the paper, we will refer to them as the speed-time, speed-time (going faster implies taking less time), distance-time (going farther takes more time), and speed-distance (going faster implies getting farther) resources.

\section{EMPIRICAL SUPPORT FOR THE MODEL}

The model of students' thinking that we have described is fairly basic. It states that students have three conceptual resources (among many) for thinking about the interrelations among speed, distance, and time that influence the way they think about a variety of situations involving motion. These resources are elements of students' knowledge that help them to make predictions and comparisons about moving objects and that may be used appropriately or inappropriately depending on the context. In the model, we assume that any one of these resources may not only be activated on its own but may also be combined with other resources to think about a given physical situation.

We present two types of data that support the utility of our particular model for describing students' thinking about motion. The first is a brief account of qualitative data taken from students' responses to a question about the motion of a mass attached to a spring. The second is an extended discussion of quantitative data resulting from an experiment intended to measure variability in student's performance with two questions about objects moving under the influence of gravity.

\section{A. Responses to simple harmonic motion}

At ASU, we observed that many students' responses to questions we posed about simple harmonic motion could be accounted for in terms of our model as well. These students had all recently completed a laboratory on simple harmonic motion, in which they investigated the dependence of period of oscillation for a mass attached to a spring on various factors such as amplitude, mass, and spring stiffness. About 100 students enrolled in an algebra-based physics course were asked to reason about the time taken for a mass attached to a spring to reach its equilibrium position after being released at various distances from equilibrium. One possible explanation for a correct answer to this question is that the time needed to pass through equilibrium is one-quarter of the period and that this period is independent of amplitude. Fewer than $20 \%$ of the students provided an explanation based on the amplitude independence of period. We found that most the answers and explanations were consistent with the resources in our model.

In an interview about this situation, a student predicts that it takes longer if the spring is stretched more:

"I just know the farther you are away, the longer it takes to get there." 
This statement is consistent with the distance-time resource. The students' attention seems to be solely on how the distance changed, suggesting to us that at this moment he is probably not attending on how the force changes with distance. The use of the word "just" suggests that the student thinks he is expressing an obvious idea-one not needing further explanation. ${ }^{14}$

Another student comes to the opposite conclusion-that the farthest one will take the least time:

"The one at the greatest distance has the greatest velocity, so it will take the least time."

This statement suggests a reliance on the speed-time resource. The response may implicitly include ideas that the student has for understanding properties of springs. diSessa ${ }^{9}$ described springiness as a p-prim, ${ }^{15}$ perhaps used in this case to conclude that springs stretched farther pull back harder. Other students' statements from written problems also seemed to indicate this kind of reasoning:

"Farther the distance, the greater the force, and the faster it will snap back."

The resources in our model can be combined (in some cases with additional ideas about springs) to give explanations for the correct answer. We might think of the examples below as a "compensation" between the speed-time resource and the distance-time resource. In the first response, the second mention of distance suggests that the student believes that extra distance is making up for the effect of the increases in velocity and acceleration.

"As distance increases so does the velocity and acceleration but so does the distance."

"They are equal because as the spring gets pulled farther and farther it stretches more. When released it will snap back at the same time."

Our simple model does not contain all of the knowledge that students bring to bear on the spring question. The inclusion of additional resources for thinking about springs and simple harmonic motion as part of responses to this question is to be expected. As we will see in Secs. IV B and IV C, students also seem to rely on additional intuitions concerning

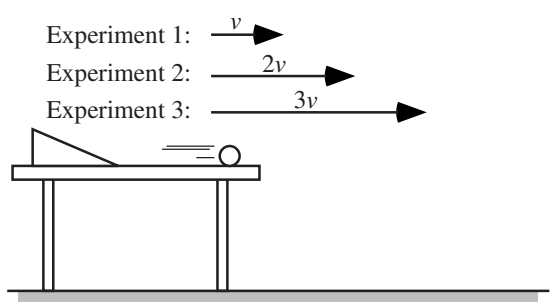

In 3 separate experiments, a student launches a ball horizontally from a table. The ball leaves the table with a speed $v$ in the first experiment, with a speed $2 v$ in the second experiment; and with a speed $3 v$ in the third experiment.

Rank, from greatest to least, the time that it takes the ball to travel from the table to the floor for the three experiments. Explain how you determined your ranking.

FIG. 1. Projectile question: speed cuing. effects of gravity when thinking about motion questions involving gravity.

\section{B. Students' performance on projectile motion tasks}

We suggest that our simple resource-based model helps account for the observed variations in distributions of student responses for the questions that were asked at ASU by supposing that students have variety of different resources for thinking about this situation in the first place. However, there were significant variations in the style of those questions (quantitative versus qualitative, ranking task versus comparison task, etc.) that complicate an analysis of the effect of the difference in prompt. In addition, the surveys were administered during different semesters taught by different instructors who sometimes used different textbooks or curricula. Because we suspected that the variations in cuing (speed or distance) of the tasks were the most significant contributor to differences in student performance, we decided to conduct a more controlled experiment with students from two sections of an algebra-based introductory course at the University of Maryland.

The questions shown in Figs. 1 and 2 were designed to be as close as possible to one another in format, wording, and style. In one section they were administered on the first day of lecture, and in the other they were administered on the second day of lecture. Almost all students who answered had taken and passed the first semester course (mechanics), the previous semester in one of the two sections of that course. The instructors for mechanics had both covered material on projectile motion and both had demonstrated and discussed the outcomes of "shooter-dropper" demonstrations ${ }^{16}$ with their classes as part of instruction. Students in both sections also had completed a laboratory in which they used equations of motion to predict where a projectile launched from a table would land.

The correct answer to both questions is that the time to reach the floor in all three cases is the same. One can arrive at this answer by reasoning that since all the balls fall from the same height, with the same initial vertical velocity and with the same vertical acceleration, the balls must reach the

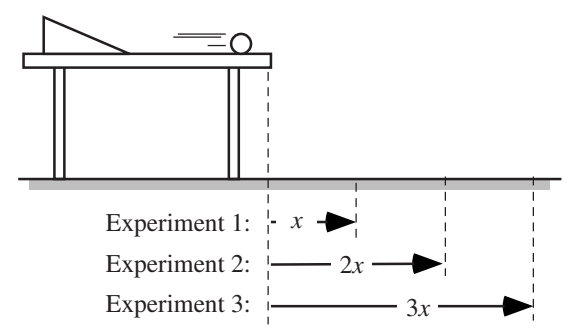

In 3 separate experiments, a student launches a ball horizontally from a table. The horizontal distance that the ball travels before hitting the ground is $x$ in the first experiment, $2 x$ in the second experiment, and $3 x$ in the third experiment.

Rank, from greatest to least, the time that it takes the ball to travel from the table to the floor for the three experiments. Explain how you determined your ranking.

FIG. 2. Projectile question: distance cuing. 
floor at the same time independent of their horizontal speeds or landing positions.

In each section we alternated versions of the survey before distributing them seat by seat. In total, the distancecuing survey was administered to 165 students, and the speed-cuing survey was administered to 160 students. Students were given about $15 \mathrm{~min}$ to complete the survey (including a second question described in Sec. IV C). Students did not include their names on their responses. Since there were no statistically significant differences in the distribution of responses between the two different sections, we have combined the results.

Based on our model and initial (ASU) results, we hypothesized that students given the speed-cuing version would be more likely to answer that the fastest ball would take the least time. In addition, we expected to find evidence in students' explanations that they were relying on the speed-time resource to arrive at this answer. On the other hand, students given the distance-cuing version would be more likely to answer that the ball that traveled farthest would take most time, and they would be more likely provide an explanation indicating they were attending to the idea that going farther implies taking more time.

\section{Student responses}

Over all, the number of students answering correctly was $62 \%$ for the speed-cuing question and $60 \%$ for distancecuing tasks. ${ }^{17}$ The different cues did not affect student performance in terms of correctness but rather affected the distribution of incorrect answers. A very small number of students (about 3\% on the speed-cuing version and $1 \%$ on the distance-cuing version) gave ranking answers that were incorrect but were not either experiment $1>$ experiment 2 $>$ experiment 3 or experiment $3>$ experiment 2 $>$ experiment 1 . These responses were not included in our analysis.

We used a chi-squared test of independence to determine that there is a significant difference in the distribution of students' responses between the two survey formats, $X^{2}(2, N=318)=21.1, p<0.001$. This difference is almost entirely in the distribution of incorrect answers (Fig. 3). The results are consistent with our hypothesis regarding how students would answer and also with the pattern observed in the ASU data: students given the speed-cuing survey were as likely to answer that the faster objects take less time (19\% of total responses) as that faster objects take more time (17\%) and students taking the distance-cuing survey were more likely to answer that more distance implies more time $(33 \%)$ than more distance implies less time (6\%).

The results naturally lead to speculation about the asymmetry of the results: about as many students taking the speed-cuing survey answered that the fastest object took the most time as those answering that the slowest object took the most time. On the other hand, only a few students taking the distance-cuing survey reasoned that the longer distances took less time. One possible interpretation is that attending to the effects of distance on the time of flight is a more common approach or more easily cued resource in general. Other reasons for this pattern are suggested in Sec. $\mathrm{V}$ and concern

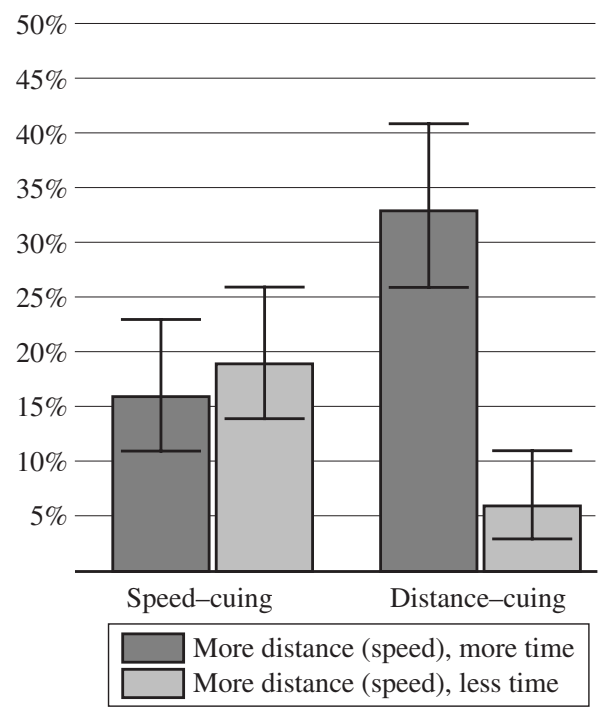

FIG. 3. Distribution of incorrect answers for projectile motion questions with $95 \%$ confidence intervals.

some nonkinematics intuitions students bring to bear on the problem.

\section{Student explanations}

Students were also asked as a part of the survey to explain their reasoning. These written explanations provide us with artifacts of students' thinking that are crude and limited indicators of the actual or complete thought processes in which they engage. They also are likely to reflect students' perceptions of what constitutes an appropriate physics explanation. Our analysis of the differences in the patterns of these responses is informative for the purpose of evaluating the plausibility of our model.

Students' explanations for the projectile motion question were coded for the type of reasoning they likely represented. Inter-rater reliability was assessed between two raters for a sample of 64 student responses, which resulted in $94 \%$ agreement. All disagreements were with regard to whether a particular explanation was clear enough to be coded as one type of reasoning or whether it should be coded as incomplete, in which case it was coded as "other." Students providing no written record of their reasoning (less than $7 \%$ of total) were removed from further analysis. Therefore, percentages given below are of those students who provided an explanation. In this section we focus our discussion on students' explanations consistent with the intuitions in our model. Other explanations given by students are discussed in Sec. V.

a. Distance-time resources. For the distance-cuing task (Fig. 2), 90\% of the students answering that longer distances take more time provided explanations consistent with the distance-time resource:

"Common sense, you would think No. 3 takes the longest because it travels the most distance."

Other students' responses seem to point to a general idea of proportionality between time and distance: 
"Time is proportional to distance. As the distance increases from a certain point, the time to reach that distance increases the further away the ball gets from its initial starting point."

Students given the speed-cuing task also provided explanations consistent with this intuition, often reasoning indirectly through the intuition that going faster implies going farther:

"The faster the ball leaves the table, it means it travels farther. Because it travels farther, it will hit the ground at a later time."

Of all students who answered that the faster balls took more time in the speed-cuing task, 50\% incorporated elements of both speed-distance and distance-time resources in their explanations. Generally, the explanations given by students support our specific model in that almost all of the students taking the distance-cuing task who answered that the longer distance takes more time gave an explanation consistent with the activation of a distance-time resource. Half of the students who answered this way with the speed-cuing task gave explanations consistent with distance-time resources as well. The other half of responses suggest that other resources for thinking about motion may be involved as well, as we will discuss in Sec. V.

b. Speed-time resources. Of the students answering that the faster balls take less time, $90 \%$ gave explanations consistent with application of a speed-time resource. One student relates his idea to an everyday experience:

"It is like a pitcher throwing a baseball; if he throws it with a greater velocity, it will reach the catcher faster."

Another student answers with a more formal explanation:

"Velocity is inversely related to time. As your velocity increases, the shorter the time will be until you hit the floor."

Few students taking the distance-cuing task gave speedtime explanations. Many of those who did gave explanations indicating they reasoned indirectly with speed-distance resources. Six of the ten students who answered that the farthest distance takes the least time provided a speed-time explanation:

"The velocity must be less for 1 than for 2 and 3, etc. I know this because it travels a small distance. The smaller the velocity, I assume that it took a longer time."

"It will take the ball the shortest amount of time to $3 x$, then $2 x$, and then $x$. The speed of the ball would have to be greater in order for it to go farther, so it will take less time for it to go the farther distance."

Consistent with our model of thinking, almost all students answering that the longer trajectories took more time gave explanations consistent with distance-time resources, and almost all students answering that the faster balls took less time gave explanations consistent with speed-time resources.

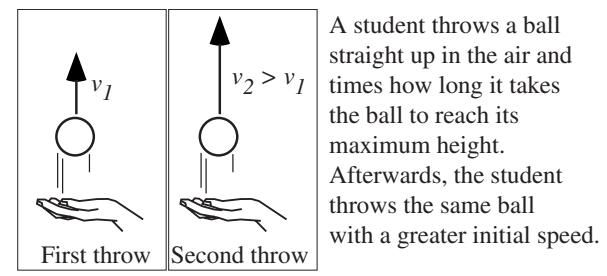

Compared to the first throw, will the amount of time taken for the ball to reach its maximum height be greater, less, or the same?

FIG. 4. Vertical toss question: speed cuing.

Many students who answered that longer paths took less time or that faster balls took more time often gave explanations which suggested they were reasoning indirectly through the speed-distance resource.

\section{Performance on vertical toss question}

As a part of our experiment, we included a question that allowed us to observe students' responses in another context for which we could use the model to make some predictions about student performance. Students who received the speedcuing question in Fig. 1 were also given a second speedcuing question, shown in Fig. 4; those who answered the distance-cuing question in Fig. 2 also were given a second distance-cuing question, shown in Fig. 5. In both versions, students were asked to compare the time for a vertically tossed ball to reach the maximum height. The distance-cuing task asks for a comparison based on two different maximum heights, while the speed-cuing task asks for this comparison based on different initial velocities. The correct answer is that a ball launched faster or reaching a greater maximum height takes more time to reach this height.

On the basis of our model we predicted that more students taking the distance survey would answer correctly because in this case the distance-time resource leads to the correct answer (since the higher toss does, in fact, take more time). Additionally, we predicted that students given the speedcuing task would be more likely to answer that the second toss would take less time compared to students given the distance-cuing task.

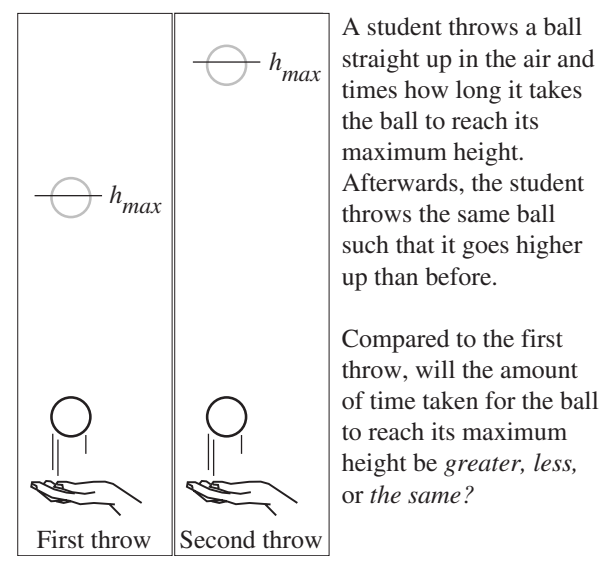

FIG. 5. Vertical toss question: distance cuing. 


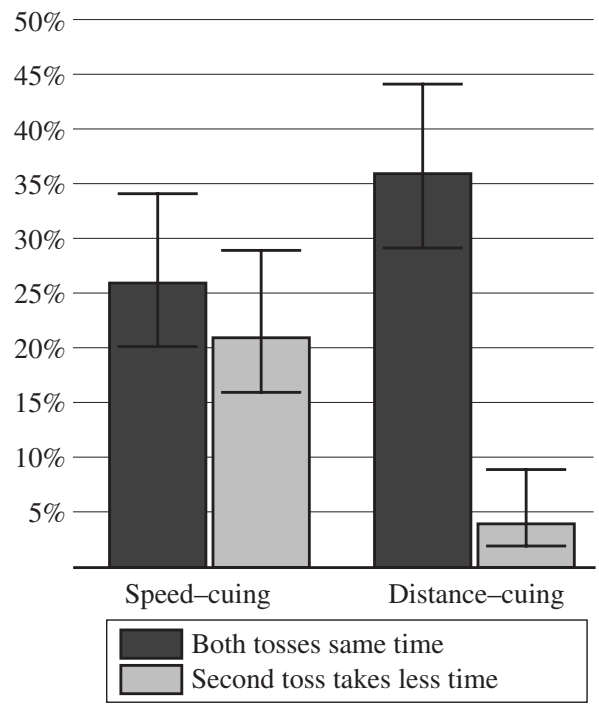

FIG. 6. Distribution of incorrect answers for vertical toss questions with $95 \%$ confidence intervals.

\section{Student responses}

The experiment supported the second one of our two hypotheses: $21 \%$ of the students taking the speed-cuing survey answered that the second toss would take less time compared to only $4 \%$ for the distance-cuing survey. A chi-squared test of independence indicates a significant correlation between which survey was taken and the proportion of students answering that the second toss takes less time, $X^{2}(1, N=309)$ $=19.6, p<0.001$. Other answers given were that the times are both the same and that the times could not be compared because they depend on information that was not provided. The distribution of incorrect answers for each version of the vertical toss question is shown in Fig. 6.

Our first prediction that students taking the distance-cuing survey would do better was not supported. Students did equally well with both questions: about $49 \%$ answered correctly for the distance-cuing question and $48 \%$ for the speedcuing question. The different cues in the vertical toss task did not seem to have an impact on the number of students answering correctly. Rather the effect of these different cues (distance or speed) is to shift the distribution of incorrect answers. However, it is important to note that the direction of this shift is not random-it is predicted based on our particular model and some additional assumptions regarding the effects of those cues.

\section{Student explanations}

Although only one of our model-based predictions was confirmed by this second experiment, students' written explanations provide us with evidence that other intuitions were used by students that are not part of our simple model. These additional intuitions suggest a refinement to our purely kinematical model, which may explain why students' performance was not entirely as predicted.

Inter-rater reliability for coding students' written explanations with the vertical toss question was $89 \%$ for a sample of 64 questions. As before, all disagreements concerned whether there was sufficient evidence to categorize an explanation as being incomplete or as representing a particular kind of reasoning.

a. Speed-time resources. For the vertical toss task, 33 of the 34 students who incorrectly answered that the second ball takes less time gave an explanation consistent with speedtime resources:

"If you do anything at a faster speed it will take less time to reach that destination."

For most of these explanations, explicit considerations for how the maximum height changes with increased speed were vague at best. Other students explicitly stated that the maximum height would be the same:

"Since distance to maximum height is the same in both cases and the velocity is increased, time must be decreased."

In these cases students apply speed-time resources with what would be an appropriate caveat-more speed does imply less time when distances are equal. However these students fail to consider other resources-perhaps speeddistance resources - that would suggest that the distance is not the same. This type of reasoning, in which quantities are inappropriately held constant (whether tacitly or explicitly), has been observed generally when students reason about situations involving multiple variables. ${ }^{18}$

b. Distance-time resources. Analysis of student explanations for correct answers on the vertical toss question provides useful insights for understanding why as many students answered correctly on the speed-cuing survey as on the distance-cuing survey. For the distance-cuing survey, nearly $70 \%$ of the students answering correctly reasoned that it would take longer to reach the higher maximum height because it is farther. Here a student gives an explanation consistent with the activation of a distance-time resource:

"The time is greater because it travels a greater distance."

However for the speed-cuing survey, only $30 \%$ of students giving a correct answer explained that the object thrown faster would go higher and thus take longer to reach its maximum height - a pattern indicative of linking speeddistance and distance-time resources together:

"Common sense tells me that the faster ball should take longer to reach the maximum height because it will travel a farther distance."

"The harder the student throws the ball the higher the maximum height will be, thus taking longer for the ball to reach it."

This was the pattern we expected based on our model: application of distance-time resources in this case leads to a correct response.

c. Resource for thinking about the effects of gravity. Although we expected that the speed cues would primarily lead to the use of speed-time resources for this problem, the cues 
seemed to also activate resources for thinking about the effect of gravity on an object's speed. A majority of students $(70 \%)$ who answered that the faster toss would take longer explained that it would be more time because a greater speed would take longer to be "overcome" by gravity.

"The acceleration due to gravity has to slow something down that's going faster, so it will take longer."

"A little more time, since it is thrown with greater upward speed in the $y$ direction, $g$ will have to work harder to bring $v$ back to 0 at the maximum height."

While this form of reasoning is not accounted for in terms of the three kinematics resources we have described in our model, it also expresses an intuitive idea about the motion of objects. Here students seem to be reasoning in a productive way about the effects of gravity. The inclusion of this idea as a resource for thinking about motion may help us to understand why students performed equally well in both versions of the vertical toss task. It is consistent with our model that the speed-cuing task would likely cue resources like more speed takes longer to be overcome by gravity than the distance-cuing task, for which only $20 \%$ of the responses indicated this kind of reasoning. ${ }^{19}$

Although our simple kinematics model was insufficient to accurately predict all features of student performance with the vertical toss tasks, we learned a great deal about the strengths and shortcomings of the model in characterizing students' responses on this question. As with the simple harmonic motion question, we observe students using these kinematics resources along with other more context-specific resources. For this task, students' explanations suggest that an intuition like gravity takes a longer time to overcome more speed played a role in their thinking along with distance-time, speed-time, and speed-distance resources. Generally, the experiment with the vertical toss questions supports our prediction that speed cues increase the number of students answering that the faster object takes less time. Students' explanations further support the claim that students answering this way were reasoning with speed-time resources. Our second prediction that more students should answer correctly with the distance cues was not supported. By examining students' written explanations, we find evidence that students bring additional resources for thinking about gravity that may account for this fact. As described in Sec. V, the results of this experiment led us to consider additional modifications to our model of student reasoning in a manner consistent with our theoretical framework.

\section{REFINEMENTS TO THE MODEL}

Additional insights into students' thinking about motion and gravity can be found by comparing and contrasting students' responses to the projectile motion and vertical toss tasks. In this section we describe how a close examination of students' explanations helped us to better understand students' thinking and to refine the model.

\section{A. "More speed implies more time" reasoning}

On the speed-cuing task presented in Fig. 1, students answering that the fastest object takes more time represented about half of all incorrect answers. Of those answers, we could only identify half of the responses as indicating students were thinking explicitly about distance-time resources as described above. The other half represented a variety of different statements claiming that more speed implies more time, which is not explicitly a resource in our kinematics model. ${ }^{20}$ We have already described one way in which this response can arise from our model-first a student reasons that the faster object travels farther and then concludes that it must have taken longer because it went farther. It seems likely that students reaching the conclusion that more speed implies more time could have been thinking in this manner and provided no written record of it. However, some students' responses are not easily accounted for with the threepiece kinematics model we describe:

"The first experiment will take the shortest amount of time; it drops to the floor from the table-however the other two with higher velocity will take longer because they will fall more gradually."

" $3 v$ will take the longest because (more) velocity will carry it further and help resist gravity."

These statements are fairly consistent with the interpretation suggested by McCloskey ${ }^{21}$ that students bring with them naive theories of motion that are quite similar to medieval impetus theories. While in our study, this kind of explanation did not emerge as a dominant pattern, they did show up more often in the speed-cuing questions than in the distance-cuing questions. With the vertical toss question, we suggested that an intuition like more speed takes longer to be overcome by gravity seems to play a role in some students' thinking. ${ }^{22}$ This resource, which was an appropriate one for reasoning to the correct answer in the vertical toss task, might also account for the incorrect responses like the two above for the projectile motion question. Inappropriate use of this intuition to the projectile motion question, without taking into account how the direction of the object that is thrown affects the time of flight, might lead some students to the answer that it should take more time to reach the floor with increased speed off the edge of the table.

\section{B. Compensation arguments}

Correct answers to the projectile motion task can be constructed by combining two of the three resources and arguing that one effect "compensates" for another. Written explanations for the projectile motion task that suggested that students were constructing compensation arguments represented only about $20 \%$ of the correct answers in the speed-cuing task and about $15 \%$ in the distance-cuing tasks. A student taking the speed-cuing survey writes the following:

"The ones with a faster velocity will travel faster and go farther, so it will take the same amount of time."

An example from a distance-cuing survey is as follows: 
"If the ball travels further then that means its rate was greater. Therefore the time for each to reach the ground from the table is equal."

For the vertical toss tasks, compensation arguments were given as an explanation by three-fourths of all students incorrectly answering that the time to reach the maximum height is the same for both tosses. Implicit in the example below is a distance-time resource compensating for a speedtime resource:

"They will both reach the maximum height at the same time. The second ball has a greater velocity so it will end up traveling farther and having a greater max height. This will balance out the speed of the two balls since the faster one has to travel a farther distance, and the times will end up being the same."

The form of the compensation argument seems to suggest that students are attending to relationships between all three kinematic quantities. This is in contrast to students reasoning with distance-time or speed-time resources alone, who appear to be using only a single relationship to reason about two of the quantities.

\section{Common feature arguments}

For the projectile motion tasks, the vast majority of students giving a correct answer included written explanations pointing to some aspect of the vertical motion (the height, the acceleration, or vertical component of velocity) that is identical in all cases. Often these students constructed compensation arguments and pointed to some common feature of the vertical motion.

Correct answers and responses to the projectile motion questions can be the result of students having memorized appropriate explanations. Alternatively, from the resources perspective we have adopted, these arguments might be viewed as refinements of the basic ideas expressed by the resources to include attendance to the independence of vertical and horizontal components of motion. (This is only one plausible explanation for these responses based on the limited information about student thinking available in the explanations.) One student's response is consistent with application of a distance-time resource to just the vertical direction:

"Because the table height is constant, the time to fall in all the three cases is the same."

Another student response suggests application of a speedtime resource to only the vertical direction:

"Equal when they hit the ground because their initial vertical velocities are the same."

Other explanations suggested that students might only be attending to the constancy of the acceleration due to gravity and do not seem to take kinematics into account. Although this type of reasoning led to correct answers for the projectile motion tasks, it led many students to incorrectly answer that the times were also the same for the vertical tosses. For these students the fact that gravity acts the same on all bodies leads them to conclude that the time is the same. ${ }^{23}$ Here is a student response from the vertical toss task:

"Time is the same with both balls because acceleration due to gravity acting on the balls is $9.8 \mathrm{~m} / \mathrm{s}^{2}$."

Our analysis of student reasoning in both of these physical situations has led us to consider two additional resources students have for thinking about gravity: gravity acts the same on all bodies and gravity takes longer to overcome greater speeds. While the former resource seems appropriate for reasoning about the projectile motion task, it leads to incorrect answers for the vertical toss tasks. The latter resource is productive for thinking about the vertical toss tasks but contributes to thinking that led to incorrect answers in the projectile motion task.

\section{Variability in individual student responses}

Even within a single student's response we sometimes see evidence of reasoning on the fly, with choices made from among a variety of possibilities. In some instances, students who gave the right answer initially gave different responses consistent with the activation of just speed-time or distancetime resources. A few students seemed to reconcile the "right" answer in such a way as to also preserve other intuitive answers. Still others explicitly gave two answers. On some papers, erased or crossed-out responses are different from the answer that is ultimately given. In one such case a student changes their answer from the correct one to an incorrect one:

"The faster it is moving the further it goes and the less time it takes to drop."

However, it was more common for students to change an incorrect answer to a correct one:

"All of the balls will hit the ground at the same time. The force of acceleration down is the same in all three cases."

In the following example a student provides two answers-one based on "common sense" and another based on an "educated guess."

"Using common sense the shorter the distance traveled the shorter the time, however, as distance increases so does $v$ proportionally, so therefore I would venture an educated guess that they are equal."

Other interesting examples came from two students who combined two different answers by claiming that the time remains the same for the vertical direction but changes along the horizontal direction.

"Even though the $y$ component times for all three experiments are the same, the $x$ component times are all different because of their different velocities."

"The faster the launch speed, the more time the ball will spend going horizontally, but they will all spend equal time going vertical."

These type of responses provide support for a knowledgein-pieces approach to understanding individual students' 
thinking about motion since they run counter to the possibility that the variance can be accounted for in terms of multiple distinct naive theories (e.g., a different flavor of misconception for each combination of answers across the two problems and cuing sets). We are only able to gather evidence of students considering multiple intuitions when they provide such written artifacts. However, it seems implausible that this dynamic is not going on within the minds of at least some of the students who do not write about it. How students come to decide among competing intuitions for thinking about this problem is certainly an interesting research question but is beyond the scope of our data.

The results of the experiment we conducted support certain aspects of the kinematics model we described in the beginning of this paper, while other student answers and explanations suggest that other resources for thinking about motion are also important to consider. Some of the students' reasoning which made little sense in one problem seemed perfectly appropriate when applied to the other problem. This is the case for reasoning that more speed takes longer to be overcome, which seems sensible for the vertical toss question but not the projectile motion question, and also for the case for gravity acts the same on all bodies, which seems like a sensible explanation for the projectile motion task but not the vertical toss question. The structure of our experiment allowed us to look at students' reasoning about motion in a few different contexts, allowing us to better understand the nature their thinking in a way that neither single context could have provided.

\section{SUMMARY AND DISCUSSION}

The experiment described in this paper was intended to test the hypothesis that students' answers to some physics questions are based on a small set of resources for thinking about motion that are common to all students. The particular model of students' knowledge we describe consists of resources that we believe students can use to think about a variety of different situations. In this paper, we describe how this model can be used to account for students' thinking about simple harmonic and projectile motions. In this model we assume that the specifics of how questions are posed influences which of the many resources are cued. Our data show that different cues included in two questions about a single physical situation resulted in variations in the distribution of student answers. These variations are consistent with predictions we made regarding the effects of cues (for example, that distance-cuing questions should be more likely to elicit an answer based on a distance-time resource). Moreover, individual written explanations were consistent with the cued activation of the three resources we included in our model. Written responses also indicate that some individual students explicitly consider multiple intuitions when responding to the questions.

We emphasize that the observed variation in student responses is not consistent with an assumption that students had singular stable beliefs or misconceptions related to the time of various trajectories. If students had an incorrect conception about how long it would take for the balls to hit the ground, then we would expect to be able to elicit the same incorrect response whether we asked the question with one cue or another. Our results suggest that for at least some students, the response is a measure of what happened at the time that the question was asked and is not an indication of a previously held conception. The ultimate aim of this paper is not to establish the inadequacy of models which characterize students' thinking about trajectories in terms of naive theories but rather to provide an example of how an explicitly manifold model of students' knowledge can be used to shed light on the variability and context dependence of students' thinking. While we are certainly not the first to point to the variability of students' thinking about trajectory to suggest that student responses are constructed "on the fly" based on contextual cues, ${ }^{24}$ we believe our contribution to research on students thinking lies in our use of an explicit model to connect this empirical variability to specific theoretical cognitive structures.

It is likely that there are other physics topics for which the hypothesis that students have difficulties or misconceptions is better supported by data and for which the pattern of responses to such questions may not appear to be strongly influenced by the specifics of the question itself. It may be useful to employ a resources framework for some topics and a different framework for other topics, as diSessa ${ }^{25}$ suggested, or it may be possible to account these results using a resource model that explains the selection of a consistent set of resources under certain circumstances. ${ }^{26}$ However, we believe that our results are much more easily explained using a resources model than they can be explained through any assumption about a priori student conceptions.

The perspective we have adopted in order to account for the variations we observe has implications for what it means to learn physics. It suggests (as others have ${ }^{27}$ ) that the transition from novice to expert in physics cannot be understood only in terms of gathering more pieces of knowledge or in terms of replacing incorrect bits of knowledge with correct ones. We suggest that many of students' correct answers can be understood as the activation of these same intuitions applied in different ways. A more complete model for learning that is consistent with a resource framework would likely include an account of mechanisms ${ }^{28}$ that serve to coordinate the use of resources in ways that effectively deal with variations in context. The lack of such an account is a major shortcoming of our model as we have described it. Although we have identified some elements of students' knowledge and have described a few ways that students often combine resources in a single context-to form a compensation argument or to string together a few resources in a chain of reasoning, for example-we offer no broader account of order, coherence, or consistency in students' ideas. Nor do we describe any "control mechanism" that determines when and why one resource is activated instead of another (beyond passive cuing). Coordination classes of diSessa and Sherin ${ }^{29}$ and Wittmann's resource graphs ${ }^{30}$ are two examples of theoretical tools for understanding and representing this aspect of knowledge structure for which our model fails to account. Thaden-Koch et $a .^{31}$ and Parnafes ${ }^{32}$ both recently reported on the utility of the coordination class concept for understanding student interpretations of motions and representa- 
tion. Such approaches may be useful for understanding the dynamics of our experiment as well but are beyond the scope of this paper.

\section{ACKNOWLEDGMENTS}

The authors are especially grateful for the contributions of
Andy Elby, David Hammer, Joe Redish, and Rachel E. Scherr. This research has been funded in part by the National Science Foundation under Grants No. DUE 05-24987, No. DUE 03-41289, No. DUE 03-41333, and No. REC 0440113.
${ }^{1}$ J. Clement, Students' preconceptions in introductory mechanics, Am. J. Phys. 50, 66 (1982).

${ }^{2}$ I. Halloun and D. Hestenes, The initial knowledge state of college physics students, Am. J. Phys. 53, 1043 (1985).

${ }^{3}$ National science foundation division of undergraduate education, Grant Nos. 05-24987, 03-41289, and 03-41333.

${ }^{4}$ M. McCloskey, in Mental Models, edited by D. Gentner and A. Stevens (Lawrence Erlbaum, Hillsdale, NJ, 1983), pp. 299-324.

${ }^{5}$ See Ref. 1.

${ }^{6}$ See Ref. 2.

${ }^{7}$ A. Champagne, L. Klopfer, and J. Anderon, Factors influencing the learning of classical mechanics, Am. J. Phys. 48, 1074 (1980).

${ }^{8}$ For a discussion about the properties commonly attributed to a model of students' understanding in a pieces model construct, see R. E. Scherr, Modeling student thinking: An example from special relativity, Am. J. Phys. 75, 272 (2007).

${ }^{9}$ A. A. diSessa, Towards an epistemology of physics, Cogn. Instruct. 10, 105 (1993).

${ }^{10} \mathrm{~J}$. Minstrell, in Research in Physics Learning: Theoretical Issues and Empirical Studies, Proceedings of an International Workshop, Bremen, Germany, 1991, edited by R. Duit, F. M. Goldberg, and H. Niedderer (IPN, University of Kiel, Germany, 1992), pp. 110-128.

${ }^{11}$ D. Hammer, Student resources for learning introductory physics, Am. J. Phys. 68, S52 (2000).

${ }^{12}$ See Ref. 9.

${ }^{13}$ See, for example, J. Piaget, The Child's Conception of Movement and Speed (Ballentine Books, New York, 1970).

${ }^{14}$ diSessa has described this property of p-prims as the principle of impenetrability (see Ref. 4, p. 121).

${ }^{15}$ See Ref. 5.

${ }^{16}$ For a description and video of this demonstration see the University of Maryland Physics Lecture-Demonstration Facility website, http://www.physics.umd.edu/deptinfo/facilities/lecdem/ services/demos/demosc2/c2-21.htm

${ }^{17}$ It had appeared based on the data collected at ASU that student performance is worse with distance cues than with speed cues. This seems not to be the case with UMD experiment, where student performed about equally as well with either survey. One possible explanation for this difference is that the questions in the UMD experiment were administered during the second semester of an introductory physics sequence, whereas with the ASU experiment they were administered during the first semester. This might also account for why student performance in general was slightly better.

${ }^{18}$ See, for example, R. Cohen, B. Eylon, and U. Ganiel, Potential difference and current in simple electric circuits: A study of students' concepts, Am. J. Phys. 51, 407 (1982); S. Rozier and
L. Viennot, Students' reasonings in thermodynamics, Int. J. Sci. Educ. 13, 159 (1991).

${ }^{19}$ One may interpret this intuition as being a specific instance of the more abstract p-prims such as dying away, overcoming, and Ohm's p-prim. We do not believe, for the purposes of this paper, that such a decomposition is particularly illuminating.

${ }^{20}$ For a detailed discussion about the nature of more speed implies more time reasoning among children, see I. Levin, Interference of time-related and unrelated cues with duration comparisons of young children: Analysis of Piaget's formulation of the relation of time and speed, Child Dev. 50, 469 (1979).

${ }^{21}$ M. McCloskey, Mental Models (Ref. 4), pp. 299-324.

${ }^{22}$ One way of reconciling the naive theory characterization with our resource-based model is to cast the naive theory in terms of the pieces within our model. The speed-distance, distance-time, and more speed takes longer to be overcome resources are all ideas that may be viewed as components of the impetus theory. In fact, although we can distinguish between these different resources as expressed in students' explanations, there is no way to distinguish between them in students' thinking based upon their answers. Although the naive theory characterization may be adequate for categorizing the most common incorrect answer, it fails to capture the kind of variability that becomes apparent in the experiments presented in this paper.

${ }^{23}$ These kinds of statements, along with many of the common feature arguments, may also be thought of as students applying a general resource like same (or similar) conditions lead to same outcomes.

${ }^{24}$ N. J. Cooke and Breedin, Constructing naive beliefs of motion on the fly, Mem. Cognit. 22, 474 (1994).

${ }^{25}$ See Ref. 9.

${ }^{26}$ D. Hammer, A. Elby, R. E. Scherr, and E. F. Redish, in Transfer of Learning from a Modern Multidisciplinary Perspective, edited by J. P. Mestre (IAP, Greenwich, 2005), pp. 89-120.

${ }^{27}$ See, for example, J. P. Smith, A. A. diSessa, and J. Roschelle, Misconceptions reconceived: A constructivist analysis of knowledge in transition J. Learn. Sci. 3, 115 (1994).

${ }^{28}$ See Ref. 26.

${ }^{29}$ A. A. diSessa and B. L. Sherin, What changes in conceptual change?, Int. J. Sci. Educ. 20, 1155 (1998).

${ }^{30}$ M. C. Wittmann, Using resource graphs to represent conceptual change, Phys. Rev. ST Phys. Educ. Res. 2, 020105 (2006).

${ }^{31}$ T. C. Thaden-Koch, R. J. Dufresnse, and J. P. Mestre, Coordination of knowledge in judging animated motion, Phys. Rev. ST Phys. Educ. Res. 2, 020107 (2006).

${ }^{32}$ O. Parnafes, What does "fast" mean? Understanding the physical world through computational representations, J. Learn. Sci. 16, 415 (2007). 